\title{
Evolution of Smart Meters (AMI) and Its Role to Make the Power Distribution Sector More Viable and To Extend Quality in Services towards Customers
}

\author{
${ }^{* 1}$ Damodara Nambeti, ${ }^{2}$ S.Varada Rajan \\ ${ }^{1,2}$ S. V. University College of Engineering, SV University, Tirupathi. \\ Email:damodara11@gmail.com,varadasouri@gmail.com
}

Received: 06th April 2020, Accepted: 05th May 2020, Published: 30th June 2020

\begin{abstract}
As a contemporary approach, nowadays the power distribution system has been working in new regime by using IT and ICT applications, to reduce the power supply-demand gap and to increase reliability of power supply which is the challenges of present power distribution sector. To achieve these, smart meter (AMI) solution is one of the best options since real-time data can be captured from remote end through bi-directional communication using GPRS/PLCC/RF technologies. The smart meters are designed to perform certain functions, stores data and communicate according to specified standards. As a part of Smart Grids, the AMI solution will provide bench mark benefits. This paper proposes award-winning "RF mesh" RF communication solution comprises of RF Module, Gateways and HES/ NMS for aforesaid requirement and solution architecture for RF mesh platform including the RF mesh canopy and RF mesh server at customer end.
\end{abstract}

\section{Keywords}

Smart Meters, Advanced Metering Infrastructure, Head End System (HES), Meter Data Acquisition System (MDAS), RF Technology, Meter Data Management System (MDMS).

\section{Introduction}

Smart meter plays a key role in the modernization society for many requirements across the globe. There is huge demand causes to power supply disturbances and outages have substantial negative impact on the quality of lives. To overcome the discrepancies at the consumer side requires advanced management of power distribution, high quality of supply and reliable system at end user side. To the demand management at the private low voltage customers or prosumers [15] and the utilities, the metering data should be acquitted and available for future.

The smartness at the meter infrastructure verifies at the Distribution System Operator (DSO) reading the metering data remotely, therefore to avoid the conventional data recording and estimation at consumer level, AMI based smart meter is proposed[1]. The metering system should operate and control by the utilities for security reasons, the provision provided at the end user level to check the consumption data and to complain the issues. This smart meter is placed at any one end of the network and the other side of the network is placed with the Head-End System (HES) at utility. The smart meter energy flows from or towards electric network should recorded as shown in Fig.1. And that data can communicate to the HES using an AMI [2-3].

Advanced Metering Infrastructure

This metering infrastructure supports the utility by managing their resource and business process effectively and efficiently. Its bidirectional data flow assessment between end users and utilities provides intelligent management, accurate maintenance, easier, proper additions and replacement of utility assets which results in better power quality.

Mainly AMI contains three basic components are smart meters at user end, bi-directional communication path between end user and utility, automated software and operation center for data processing. The basic functionalities of the smart metering system defined [1] are: remote meter data reading at configurable intervals (push/pull), time of day (TOD)/TOU metering, pre-paid/post-paid functionality net metering/billing, remote disconnection/ reconnection, alarm/event detection, notification and reporting and remote load limiter and connection/ disconnection at defined/on demand.

In addition to above the following functionalities are also required as i) Field replaceable modular communication module (shall be bundled in meter housing ii) Pairing with communication module iii) Geo location identification iv) Time synchronization.

\section{Major Components of AMI Smart Metering System}

a) Smart Meters: These electronic digital energy meters have the possible bi-directional communication and import \& export energy flow measurement.

b) Communication Network: It consists of two segments - the local area network (localized to meters in the field) and the wide area network (LAN to central station) - this communication infrastructure enables bi- 
directional communicating of data between smart meters and utility. The selection of metering system depends on the applications and various ways of field-based communication infrastructure.

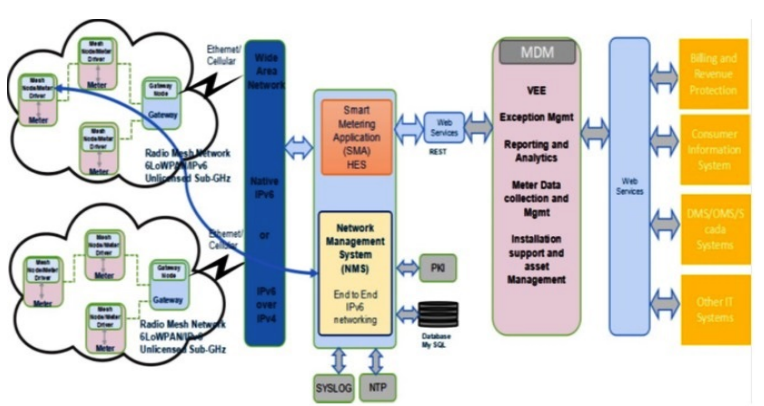

Fig. 1: AMI Solution Architecture

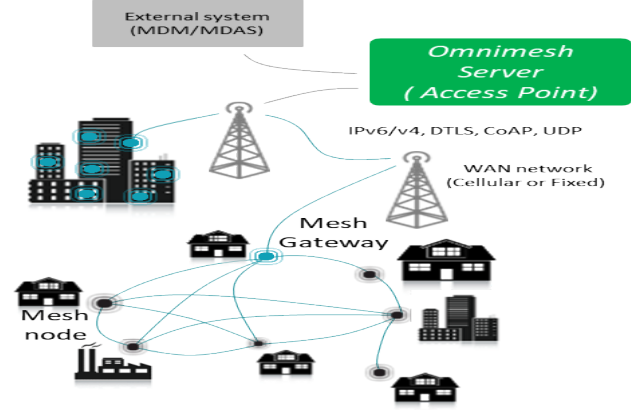

Fig. 2: RF Mesh Network

c) Head End System (HES)/ Meter Data Acquisition System (MDAS): To acquire data from different end points and monitor them automatically from remote. To acquire data from different end points and monitor them automatically from remote. This system is to favor of responding to the role of RF mesh communication solution provider and shall provide the RF mesh module, gateways to form the RF Canopy and HES software for reliable and secure data transfer from smart meters to HES system. The metering system offers a credible plan for delivering communications solutions that are: competitively priced, fully functioning, interoperable, and standards based. It is excellent in radio mesh technology and field deployment allows it to offer a fully compliant solution. This is ideally suited to smart metering and provides the following key benefits as i)True narrow band RF mesh at 865-867 MHz, ii) Lower proportional bill of materials (cost/ manufacturing / resiliency) and ease of deployment. iii) Access point intelligence with centralized command and control. iv) Network resilience and robust operation through self-forming, self-healing algorithms, v) Efficient spectrum utilizations and channel management to enable QoS (Quality of Service) network profiling and management.vi) Adaptive noise floor monitoring algorithms and listen before talk (LBT) "good neighbor" coexistence implementations.

d) Network Management System (NMS): It is responsible for the establishment and management of all radio mesh networks, the discovery of all nodes once deployed in the field and the overall system management as well as security management.

e) Meter Data Management System: It is fully software based application which stores, validates, edits and analyze the meter reading data earlier to provide it to integration into other utility operational systems such as customer billing, load forecasting and outage management.

f) Interfaces and Integration: The meter reading modifies to enhanced automated meter reading by integrating the information and build interfacing between existing and new specific applications to be supported utility endto-end business processes.

g) Mobile Application: This application also serves purpose of In-Home Display (IHD) and web application used by consumer for monitoring usage. The AMI system should support with strong user management, administrative area management (utility administrative hierarchy like zone, circle, division, sub division etc.) and flexible tabular, graphical reporting module.

Smart meters will enable customers to take advantage of new tools to save energy and money.

h) RF Technology: With increasing demand and rising levels of data, higher spectrum frequency networks, such as cellular networks, are under high pressure. Narrowband RF mesh technology uses lower bandwidth radio frequencies that offer a robust and unoccupied alternative. Narrowband applications use considerably less power and are less spectrum-intensive than those using higher frequencies, offering more capacity at a lower cost.

i) RF Mesh: RF mesh platform is an open standards-based (IPv6, 6LoWPAN) wireless neighbourhood area network (NAN). The solution provides long-range and reliable communication for millions of smart meters in an AMI deployment. It utilizes the narrowband, license free, Sub-GHz frequency spectrum (e.g. 865-867 MHZ) for mesh networking using an inexpensive radio transceiver. The platform allows geographically distributed devices (meters) to connect to the internet / WAN (Wide Area Network) via a local gateway using Ethernet or cellular connections.

The RF mesh network as shown in Fig.2 is an always ON, horizontally scalable, self-healing, self-configuring and self-discovering network, this provides excellent penetration through obstructions, such as buildings, in smart metering deployments. The mass-management features of RF mesh solution make it easy to provide an overview and manage large deployments. The system is developed in a modular way with robust security features. 


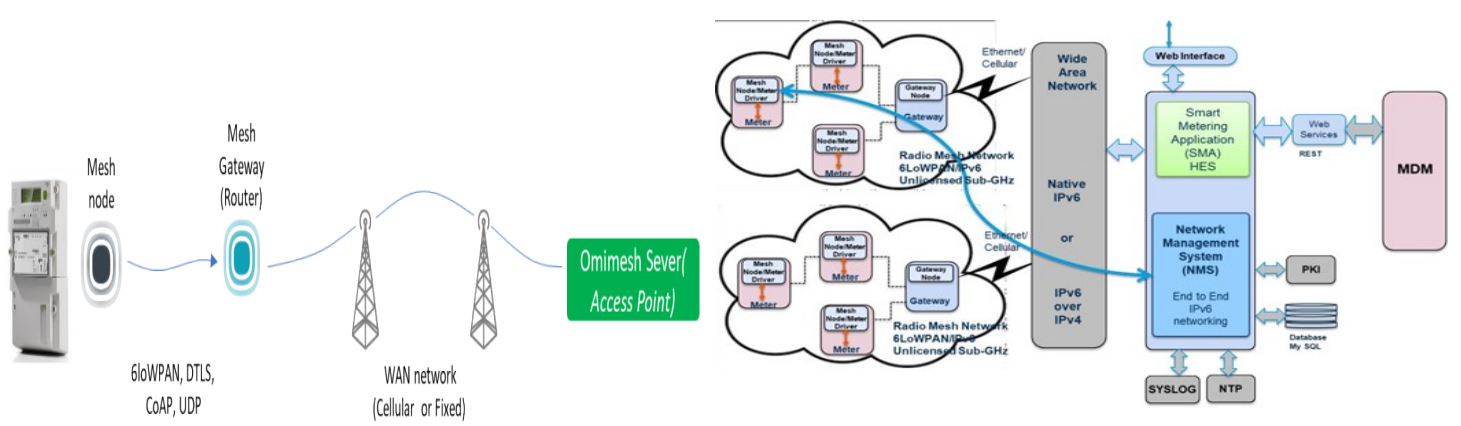

Fig. 3: Mesh Gate Way Connection

Fig. 4: End-to-End RF Mesh Solution

\section{Network Planning Study for Gateway Installation}

The network planning study is carried out for deployment of the RF canopy in a new geography. The aim for this planning is to analyse the mesh networks performance within the local geographical constraints and infrastructural builds and efficiently use the valuable bandwidth with best adaptation to the local condition.

Optimal RF network planning is essential to deploy and gracefully manage a well-balanced and stable mesh network with right number of GW routers to manage dense meter population in urban areas or sparse meter population in rural areas and achieve reliable connectivity to meters even inside buildings, hard to reach locations.

The identified installation area is sliced down into circles of $200 \mathrm{~m}-400 \mathrm{~m}$ diameter based on terrain, consumer density and meter installation type. Network planning process identify the most appropriate place to install the Gateway 200 in such a way that approximately 150 to 200 smart meters establish communication with 5-10 hops. While deciding the gateway location and number of meters to served, emphasis is given to keep some reserve capacity such that in event of a gateway coming out of the WAN connectivity ( hardware failure or cellular outage ) the node connected to the RF Mesh server can have the best probability to connect with a adjacent gateways with overlapping range .

\section{Solution Methodology to RF Mesh Nodes}

An RF Mesh Node designates a device (smart meter) fitted with an RF module running the RF Mesh Agent firmware. An RF Mesh Node joins a radio mesh network and ensures connectivity to the WAN using advanced mesh technology containing multiple RF Mesh Nodes and one Omni Mesh Gateway. Each RF Mesh Gateway provides access to the Wide Area Network and to the RF Mesh Server for all Mesh Nodes deployed in a given mesh network.

The RF Mesh Agent is part of the communication module (Mesh Node) integrated/embedded into a meter providing IPV6 connectivity. The Agent also runs the device dependent communications protocols (e.g. DLMS, ANSI) to collect data and events, trigger a connect/disconnect command, update the firmware and configuration with its attached meter.

i. RF Mesh Gateway Routers shown in Fig.3 are the focal point of Smart NAN AMI points or nodes and supports approximately 200 nodes AMI points for Smart Meters.

ii. RF Mesh Server is the access point used to send and receive information through Gateway nodes and meter nodes as shown in Fig.4. The RF Mesh Server provides overall network management and routing management for on-demand connectivity as well as scheduled readings.

iii. The RF Mesh mesh Canopy is resilient, intelligent and simple to deploy. The network nodes are selfconfiguring and support intelligent routing. IPv6 Routing Protocol for Low-Power and Lossy Networks (RPL) is used as the routing protocol by all radio nodes during discovery to allow a "topology" to be computed locally by each node. The selection by a node of its "parent" among all possible radio neighbor is a local and automatic decision made by the node itself.

\section{Salient Features \& Applications of Smart Meters}

Self-Forming- The nodes constantly try to optimize the network topology by evaluating the radio conditions to neighbouring nodes and always adapt to the best local radio conditions. Mesh nodes may decide to change parent within a mesh network or may decide to join another adjacent mesh networks. Those local adaptations do not affect the end-to-end IPv6 connectivity with back-end systems. A level of "stickiness" is configurable in the system so that each RF mesh Node will have to evaluate, if a change in the topology (intra mesh or inter mesh networks) is "worth" the transient level of instability resulting from this change. The intelligent routing allows mesh nodes to route data packets for nodes that may not be within the direct wireless.

Self-Healing, Fault Tolerance-The mesh is self-healing and fault tolerant. If a node becomes unavailable or a Gateway loses its backhaul connection, the network will rearrange itself automatically. The nodes will constantly 
try to optimize the network topology by evaluating the radio conditions to neighbouring nodes. Therefore, if a single node fails other connections will automatically be found, leading to a highly robust infrastructure with no single point of failure.

Non-Line of Sight (NLoS) Coverage-All the mesh nodes act as relays and the mesh topology eliminates NonLine of Sight (NLOS) coverage problem. The radio mesh can penetrate hard to reach locations as messages are bounced off by other nodes in the vicinity until they reach a Gateway with a suitable cellular WAN connection.

Ease of Incremental Deployment- The mesh network reach and value can be incrementally enhancing by adding additional nodes. Additional Gateways can be added to provide additional capacity in the canopy network and to extend the radio Neighbour Area Network (NAN).

This technology reduces the gap between utility and user, avoids the cyber challenges at generation, transmission and distribution networks, and provides the accurate energy flows from the system, regular recording of energy reduces the line Outages, tariffs, fault detection, distribution Losses etc., and improves the system efficiency, stability and more reliable and some more applications as shown in below Fig.5.

\section{Result and Discussions}

The smart meter parameters in real time applications may vary, such that to view the performance of meter, a $360^{\circ}$ software helps to remove the barriers between utility and end user towards billing, load profile, events and alarms, name plate details, consumption analysis etc. The 360 degree software monitoring system is shown in Fig.6. The system generates reports for meter reading, data validation, estimation, export, events, communications and logs.

\section{Communication Report For a Period of 30 Days}

In the following Fig.7 shows the procedure to analyze the communication data for the loads connected to the utility in two forms as mentioned:

\section{Typical Reports}

Export Format: The reporting tools shall offer users the option of exporting reports to disk or other computer system using a variety of standard formats.

Date Selection: The system shall provide users with the option to enter a specific date range, or to run reports using standard time scales such as today, yesterday, week to date, month to date, last seven days etc.

Data Availability: The system shall provide a mechanism to report on the time periods for which data is available or not available at a selected customer as shown in Fig.7

Analytical Reports

The system provides extensive capabilities for analytical reporting on the interval data stored in the system. Proposed solution provides mobile apps to bring mobility in to hands of the users which would help in many key areas of the solution such as survey, meter installation and replacement, monitoring and CIS.
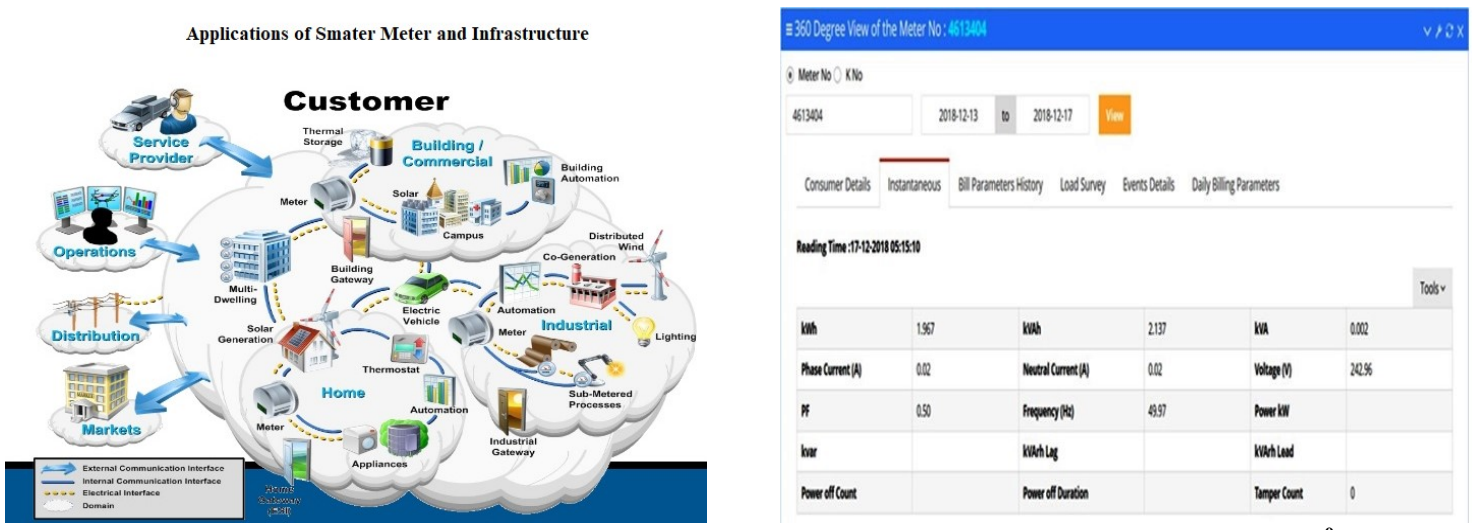

Fig 5: Applications of Smart Meter and Infrastructure Fig 6: Smart Meter Reading using $360^{0}$ Software

Some of the features are also available at consumer app as stated below and shown in Fig.8: i) consumer consumption analysis, ii) disconnection \& reconnection alerts, iii) prepaid recharge/ pay bill iv) on demand consumer read and estimation of bill, v) outage alerts

At consumer side by utilizing the smart meter mobile app, the user can access the power generation, consumption in terms of energy units and received SMS alerts regarding the tariff. By implementing the service at end user side the authorized official may also have an record and receive information about the load consumption at each point. 


\section{Conclusion}

The implementation of smart meter along with its application at consumer end extends the quality of power in service towards reduction in cost, recognition of discrepancies at both utility and end user. The functionalities and the working of a smart meter, AMI, HES and RF mesh technologies for data structure and communications are also explained by using AMI at consumer side. The smart meter application is one of the best solution to achieve and maintaining customer engagement, metering data and other related information to extend the quality of power supply in service.

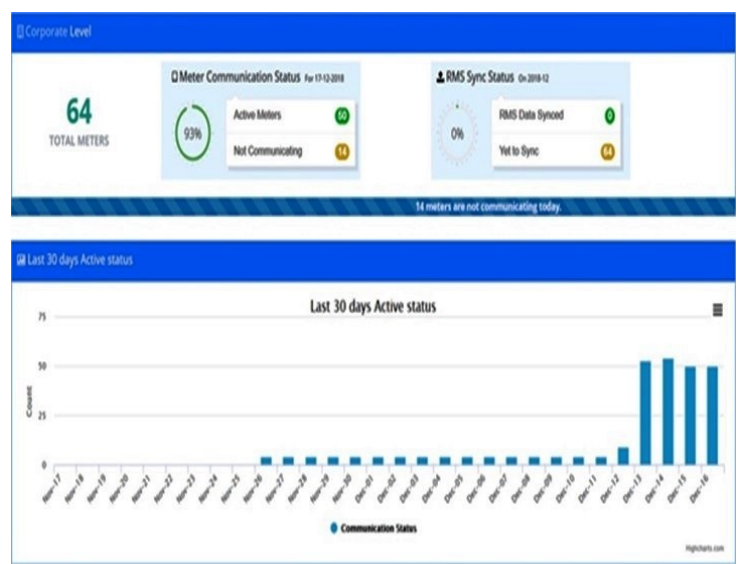

Fig.7: Meter Communication Reports for 30 Days

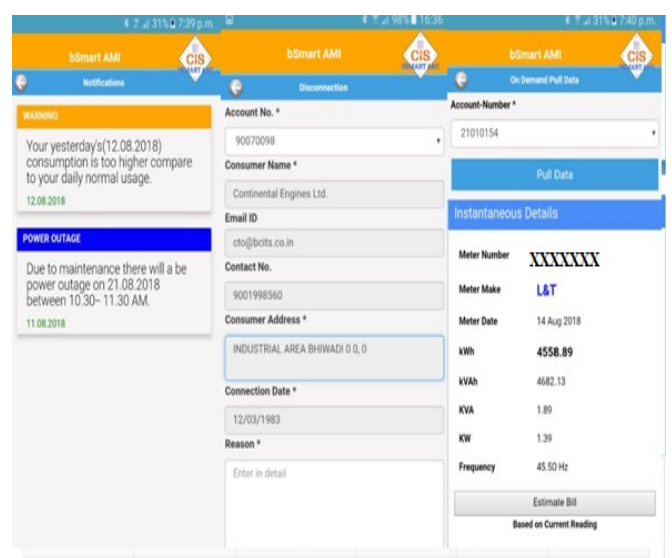

Fig. 8: Features in Consumer App

\section{References}

1. Gouri R. Barai, Sridhar Krishnan, and Bala Venkatesh, "Smart Metering and Functionalities of Smart Meters in Smart Grid - A Review” , IEEE, EPEC 2015 London, ON, Canada, 978-1-4799-7664-5/15, pp.138-145, 2015.

2. Muhammad Rizwan Asghar, Gy"orgy D'an, Daniele Miorandi and Imrich Chlamtac, "Smart Meter Data Privacy: A Survey" IEEE Communications Surveys \& Tutorials, ISSN- 1553-877X, DOI 10.1109/COMST.2017.2720195, pp.01-16, 2016.

3. Damminda Alahakoon and Xinghuo Yu," Smart Electricity Meter Data Intelligence for Future Energy Systems: A Survey", IEEE Transactions On Industrial Informatics, ISSN- 1551-3203, pp. 01-12, 2015.

4. Alessandro Pitì, Giacomo Verticale, Cristina Rottondi, Antonio Capone and Luca Lo Schiavo, "The Role of Smart Meters in Enabling Real-Time Energy Services for Households: The Italian Case", article in Energies 2017, 10, 199; doi:10.3390/en10020199, pp.01-25.

5. Krystian X. Perez, Dr. Michael Baldea and Dr. Thomas F. Edgar, "Meters to Models: Using Smart Meter Data to Predict and Control Home Energy Use" Research Gate pp.01-12, 2016.

6. Wenpeng Luan, Joshua Peng, Mirjana Maras, Joyce Lo, and Brian Harapnuk, "Smart Meter Data Analytics for Distribution Network Connectivity Verification", IEEE TRANSACTIONS ON SMART GRID, ISSN1949-3053, pp.01-08, 2015.

7. Visalatchi S, Kamal Sandeep K," Smart Energy Metering and Power Theft Control using Arduino \& GSM", IEEE, 2nd International Conference for Convergence in Technology (I2CT), 978-1-5090-4307-1, pp. 858$861,2015$.

8. Jixuan Zheng, David Wenzhong Gao, Li Lin, "Smart Meters in Smart Grid: An Overview", IEEE Green Technologies Conference and IEEE computer Society, 978-0-7695-4966-8/13, pp. 57-64, 2013.

9. R. Morello, C. De Capua, G. Fulco, S.C. Mukhopadhyay, "A Smart Power Meter to Monitor Energy Flow in Smart Grids: The Role of Advanced Sensing and IoT in the Electric Grid of the Future", IEEE sensors Journal, ISSN-1558-1748, pp.01-10, 2017.

10. Gordon Rausser, Wadim Strielkowski and Dalia Streimikiene, "Smart meters and household electricity consumption: A case study in Ireland”, Article in Energy \& Environment Vol.0(0), pp. 1-16,2017.

11. Elisa Spanò, Luca Niccolini, Stefano Di Pascoli, and Giuseppe Iannaccone, "Last-Meter Smart Grid Embedded in an Internet-of-Things Platform", IEEE Transactions On Smart Grid, ISSN-1949-3053, pp.01$09,2014$.

12.P. McDaniel and S. McLaughlin, "Security and privacy challenges in the smart grid," IEEE Security and Privacy Mag., vol. 7, no. 3, pp. 75-77, 2009.

13. N. Komninos, E. Philippou, and A. Pitsillides, "Survey in smart grid and smart home security: Issues, challenges and countermeasures," IEEE Communications Surveys Tutorials, vol. 16, no. 4, pp. 1933-1954, 2014. 
14.P. G. Da Silva, D. Iliæ, and S. Karnouskos, "The impact of smart grid prosumer grouping on forecasting accuracy and its benefits for local electricity market trading," IEEE Trans. Smart Grid, vol. 5, no. 1, pp. 402410, Jan. 2014.

15. S. Karnouskos, "Smart houses in the smart grid and the search for value-added services in the cloud of things era," in Proc. of IEEE Intl. Conf. on Industrial Technology, 2013. 